\title{
Radiative Strength Functions for Dipole Transitions in ${ }^{57,59}$ Co
}

\author{
S. S. Ratkevich, ${ }^{1}$ I. D. Fedorets, ${ }^{1}$ B. A. Nemashkalo, ${ }^{2}$ and V. E. Storizhko ${ }^{3}$ \\ ${ }^{1}$ Karazin Kharkiv National University, Ukraine \\ ${ }^{2}$ Kharkov Institute for Physics and Technology, Ukraine \\ ${ }^{3}$ Institute for Applied Physics, NASU, Sumy, Ukraine
}

\begin{abstract}
Average-resonance data on the $(p, \gamma)$ reaction on ${ }^{56,58} \mathrm{Fe}$ that were taken at proton energies of $E_{p}=1.5 \div 3.0 \mathrm{MeV}$ are used to determine the absolute values of the radiative strength functions for energies below $10 \mathrm{MeV}$. The results obtained in this way are compared with the results of the calculations that rely on the statistical approach and which take into account the temperature of the nucleus and its shell structure. Good agreement with experimental data is achieved without any variation of parameters.
\end{abstract}

PACS numbers: 21.10.Ma,21.10.Pc,23.20.Lv,25.40.Lw,24.60.Dr

\section{INTRODUCTION}

Experimental and theoretical investigations of the energy dependence of radiative strength functions for nuclei whose shells are filled almost completely or completely revealed that, if the Lorentz distributions that describe well the electric giant dipole resonances excited in such nuclei are extrapolated to the region of low energies, the resulting curve complies with experimental data neither in absolute value nor in shape. For example, an extrapolation of a Lorentzian curve to the region of low energies of gamma rays yields radiative-strength-function values that are eight times as great as the corresponding experimental values obtained in [1] for the ${ }^{59}$ Co nucleus, which has a nearly filled proton shell $(Z=27)$. Attempts undertaken in [1] to change the absolute values of the radiative strength function by varying parameters used in determining this function proved to be futile; therefore, its behavior was considered to be anomalous. On the other hand, the same authors [2] obtained data on the radiative strength function for the ${ }^{65} \mathrm{Cu}$ nucleus that were in good agreement with the extrapolation of the corresponding Lorentzian form. A deviation of the radiative strength function from the Lorentzian behavior is at odds with the well-known Brink hypothesis. In accordance with this hypothesis, primary $E 1$ transitions that are observed in radiative nucleon capture are associated with the same processes as giant dipole resonances approximated by a Lorentzian form; moreover, giant resonances built on the ground state and on excited states of the final nucleus are described in terms of the same parameters. The above deviations may suggest the nuclear-structure dependence of the radiative strength function.

The objective of the present study is to determine the absolute values of the radiative strength functions for the electric dipole transitions in ${ }^{57,59} \mathrm{Co}$ nuclei near the nucleon binding energy and to analyze their energy dependence. We determine here the relevant radiative strength functions from the averaged intensities of primary gamma transitions that proceed to individual low-lying states of the nuclei being investigated and which are excited in the $(p, \gamma)$ reactions on ${ }^{56,58} \mathrm{Fe}$ nuclei at incident-proton ener- gies between 1.5 and $3.0 \mathrm{MeV}$. The energy $Q$ of the $\left(p, \gamma_{0}\right)$ reactions on these target nuclei is $6.02 \mathrm{MeV}$ for ${ }^{56} \mathrm{Fe}$ and $7.37 \mathrm{MeV}$ for ${ }^{58} \mathrm{Fe}$. These values of $Q$ are sufficiently large for the densities of states in compound nuclei to satisfy the requirements that ensure the applicability of the statistical description. The thresholds for the $(p, \gamma n)$ reactions on ${ }^{56} \mathrm{Fe}$ and ${ }^{58} \mathrm{Fe}$ nuclei exceed 5 and $3 \mathrm{MeV}$, respectively. Owing to this, investigations could be performed over a wide range of incident-proton energies below the neutron threshold.

\section{EXPERIMENTAL RESULTS AND THEIR ANALYSIS}

Following [1], we determined the radiative strength functions in question by the method of averaging over the resonances of a compound nucleus formed upon incidentproton capture by the target nucleus. This averaging, which is necessary for effectively suppressing PorterThomas fluctuations [3] and for achieving a satisfactory statistical accuracy, was ensured by an optimal choice of target thicknesses and by a successive addition of gammaray spectra measured at different energies with a step equivalent to the target thickness. In taking an average over an interval of width $180 \mathrm{keV}$ for ${ }^{57} \mathrm{Co}$ and an average over an interval of width $220 \mathrm{keV}$ for ${ }^{59} \mathrm{Co}$, the scatter of data that is associated with Porter-Thomas fluctuations did not exceed the statistical uncertainty of measurements, which was within $20 \%$.

We used targets manufactured by electrolytically precipitating, onto a gold substrate, ${ }^{56} \mathrm{Fe}$ (the degree of enrichment was $99.9 \%$ ) in order to obtain $849 \mathrm{mg} / \mathrm{cm}^{2}$-and $1.729 \mathrm{mg} / \mathrm{cm}^{2}$-thick samples or ${ }^{58} \mathrm{Fe}$ (the degree of enrichment was $90.7 \%$ ) in order to obtain $849 \mathrm{mg} / \mathrm{cm}^{2}$ thick samples. The measurements were performed by using protons accelerated by an electrostatic accelerator to energies in the range $1.5 \div 3.0 \mathrm{MeV}$, which was scanned with a variable step equal to proton-energy losses in the target. The spectra of gamma rays corresponding to primary transitions were measured by a pair spectrometer arranged at an angle of $55^{\circ}$ to the proton-beam direction. The yields of gamma rays corresponding to direct 


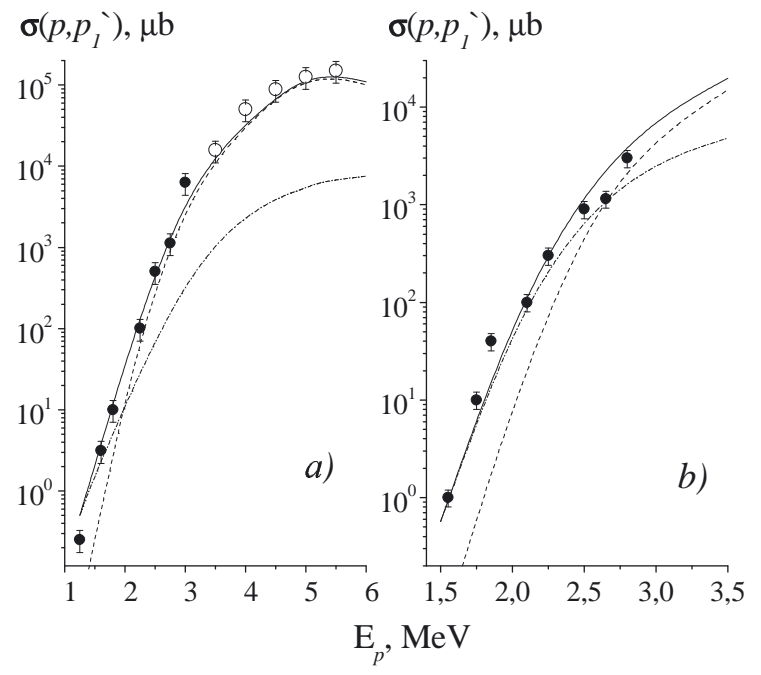

FIG. 1: Cross sections for inelastic proton scattering on $(a)$ ${ }^{56} \mathrm{Fe}$ and $(b){ }^{58} \mathrm{Fe}$ target nuclei (experimental points and calculated curves): (closed and open circles) experimental data from [11] and [12], respectively; (dash-dotted and dashed curves) contributions to the calculated cross sections from, respectively, Coulomb and nuclear scattering; and (solid curve) the sum of the Coulomb and nuclear contributions.

transitions to the ground states of ${ }^{57} \mathrm{Co}$ and ${ }^{59} \mathrm{Co}$ were also measured with the aid of a $\mathrm{NaI}(\mathrm{Tl})$ detector of dimensions $200 \times 200 \mathrm{~mm}^{2}$. The strategy of our experiment and the procedure that we used for specific measurements were described in detail elsewhere [4].

As in [1], radiative strength functions were determined here by using the fact that the radiative-strength function $\left(S_{\lambda f}\left(E_{\gamma}\right)\right.$ for electric dipole transitions can be related to the proton-capture cross section $\sigma\left(p, \gamma_{f}\right)$. With the aid of the HauserFeshbach formula, the relation in question can be reduced to the form

$$
\begin{aligned}
& \bar{\sigma}_{p, \gamma_{f}}=\frac{\pi \lambda_{p}^{2}}{2(2 I+1)} \sum_{I_{c} j_{p} l_{p}}\left(2 I_{c}+1\right) \times \\
& \times \frac{T_{l_{p} j_{p}} \times 2 \pi E_{\gamma}^{3} S_{\lambda f}\left(E_{\gamma}\right)}{\sum_{j_{p^{\prime}} l_{p^{\prime}}} T_{l_{p^{\prime}} j_{p^{\prime}}}+\sum_{j^{\prime}} \int_{0}^{E_{\lambda}} 2 \pi \rho_{j^{\prime}}\left(E_{\lambda}-E_{\gamma}\right) E_{\gamma}^{3} S_{\lambda f}\left(E_{\gamma}\right) d E_{\gamma}}
\end{aligned}
$$

where $\lambda_{p}$ is the incident-proton wavelength; $I$ is the spin of the target nucleus; $I_{c}$ is the spin of the compound nucleus; $j_{p}$ and $l_{p}$ are, respectively, the spin and the orbital angular momentum in the input channel; $j_{p^{\prime}}$ and $l_{p^{\prime}}$ are the corresponding quantities in the output channel involving proton emission; $S_{\lambda f}\left(E_{\gamma}\right)=S_{\lambda f}^{E 1}\left(E_{\gamma}\right)+S_{\lambda f}^{M 1}\left(E_{\gamma}\right)$ is the sum of $E 1$ and $M 1$ radiative strength functions for transitions from the group $\lambda$ of com-pound-nucleus states at energy $E_{\lambda}$ to the state of energy $E_{f} ; T_{l_{p} j_{p}}$ and $T_{l_{p^{\prime}} j_{p^{\prime}}}$ are the penetrability factors for protons in the input and the output channel, respectively; and $\rho_{j^{\prime}}\left(E_{\lambda}-E_{\gamma}\right)$ is the density of levels characterized by a spin $j^{\prime}$ and an excitation energy $E=\left(E_{\lambda}-E_{\gamma}\right)$. In our calculations, we took into account the correction for cross-section fluctuations of the Ericson type, which arise because of a small number of open channels, since such a correction may prove to be of importance at low energies [5]. It was assumed in [1] that, for $E 1$ transitions, the dependence of the radiative strength function on the energy $E_{\gamma}$ has the form

$$
S_{\lambda f}\left(E_{\gamma}\right)=a \frac{10^{-14}}{2 \pi} A^{8 / 3} E_{\gamma}^{k-3}\left(\mathrm{MeV}^{-3}\right),
$$

where $a$ and $k$ are parameters, whose values are fixed in fitting expression (2) to experimental data. The value of $k=4.7$ was obtained in [1] by extrapolating the Lorentzian form that describes the giant dipole resonance in ${ }^{59} \mathrm{Co}$ to the energy region under study. For the case of a direct $\gamma_{0}$ transition to the ground state of the ${ }^{59} \mathrm{Co}$ nucleus, a least squares fit of the theoretical cross section $\sigma\left(p, \gamma_{0}\right)$ to its experimental value yielded $a=1.5[1]$. The radiative strength function as determined by using the above values of the parameters $a$ and $k$ reproduces the slope of the Lorentzian curve, but the absolute values of this function differ from that which would be expected on the basis of extrapolation by a factor of $8[1]$.

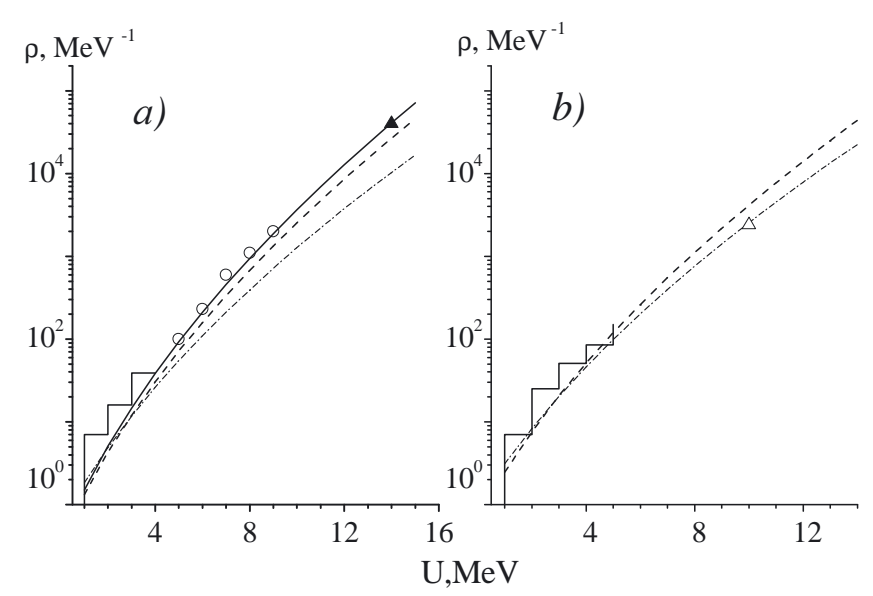

FIG. 2: Level densities in the $(a){ }^{57} \mathrm{Co}$ and $(b){ }^{59} \mathrm{Co}$ nuclei versus excitation energy: (broken lines) discrete levels established in [14, 15], (open circles) experimental data from [17], (closed triangle) experimental result from [18], (open triangle) experimental result from [19], (dash-dotted curve) results of the calculation within the back-shifted Fermi gas model with the parameter values from [20] and the nuclear moment of inertia set to half the rigid-body value, (dashed curve) results of the calculation within the back-shifted Fermi gas model with the parameter values from [20] and the nuclear moment of inertia set to the rigidbody value, and (solid curve) results of the calculation with the parameter values adopted in the present study.

In the present study, the radiative strength function appearing in the denominator on the right-hand side of (1) was parameterized either in a Lorentzian form or in that form which was obtained in the approach developed in $[6,7]$ on the basis of Fermi liquid theory. 
The quantity $S_{\lambda f}\left(E_{\gamma}\right)$ in the numerator was chosen in such a way as to reproduce the absolute values of the partial cross section that were obtained experimentally. The penetrability factors for protons were calculated with allowance for the results reported in [8-10]. The parameters of the optical potential were determined from the best fit to the experimental cross section for the reactions ${ }^{56,58} \mathrm{Fe}\left(p, p^{\prime} \gamma\right.$ ) (Fig.1), ${ }^{56,58} \mathrm{Fe}(p, \gamma)$, and ${ }^{58} \mathrm{Fe}(p, n)$ in the region of incident-proton energies below $4 \mathrm{MeV}$. The experimental cross-section values presented in Fig.1 were borrowed from $[11,12]$ for inelastic proton scattering on ${ }^{56} \mathrm{Fe}$ nuclei and from [13] for inelastic proton scattering on ${ }^{58} \mathrm{Fe}$ nuclei. For the geometric parameters of the real part of the optical potential, we chose the values

$$
\begin{aligned}
& r_{r}=1.17 \mathrm{fm}, r_{s}=1.32 \mathrm{fm}, \\
& a_{r}=0.70 \mathrm{fm}, a_{s}=0.58 \mathrm{fm} .
\end{aligned}
$$

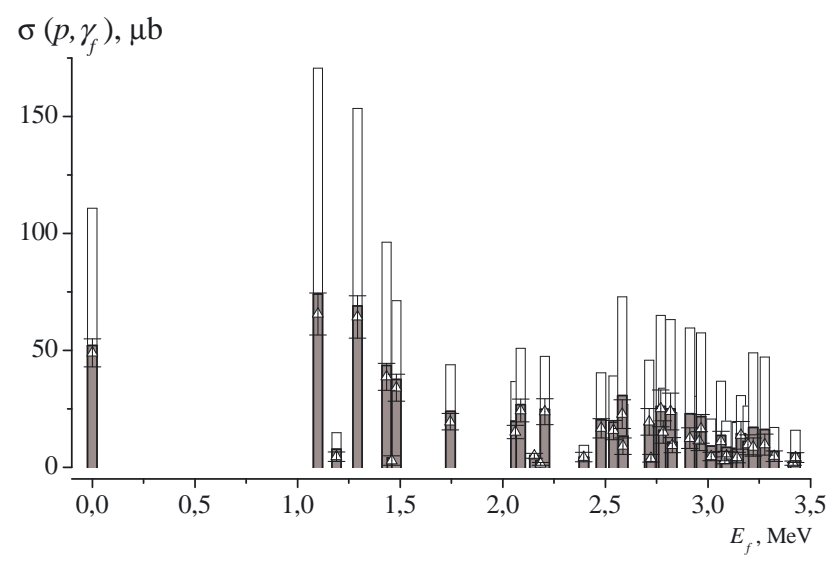

FIG. 3: $\left(p, \gamma_{f}\right)$ partial cross sections for ${ }^{58}$ Fe targets: (shaded areas of the histogram) cross sections calculated with the radiative strength functions as obtained within the statistical approach [6,7] allowing for shell effects and for the nuclear temperature, (unshaded sections of the histogram) cross sections calculated with the radiative strength functions in a Lorentzian form, and (open triangles) experimental cross sections.

With the exception of the diffuseness parameter set to a value less than that in [8], all the above values are identical to those from that study. For the real part of the potential, we took the values

$$
\begin{gathered}
V_{r}(E)=59.34-0.37 E \text { for }{ }^{56} \mathrm{Fe}, \\
V_{r}(E)=58.0-0.32 E \text { for }{ }^{58} \mathrm{Fe}
\end{gathered}
$$

for the imaginary part of the surface potential, we set

$$
\begin{gathered}
W_{s}(E)=3.85+0.72 E \text { for }{ }^{56} \mathrm{Fe}, \\
W_{s}(E)=5.6-0.25 E \text { for }{ }^{58} \mathrm{Fe} .
\end{gathered}
$$

TABLE I: $\left(p, \gamma_{f}\right)$ partial cross sections for ${ }^{56} \mathrm{Fe}$ targets

\begin{tabular}{c|c|c|c|c|c}
\hline$E_{f}, \mathrm{MeV}$ & $J^{\pi}$ & $\begin{array}{c}\sigma^{\text {exp }}, \mu \mathrm{b} \\
E=2.84 \mathrm{MeV} \\
\Delta=180 \mathrm{keV}\end{array}$ & $\begin{array}{c}\sigma_{1}^{\text {calc }} \\
\mu \mathrm{b}\end{array}$ & $\begin{array}{c}\sigma_{2}^{\text {calc }}, \\
\mu \mathrm{b}\end{array}$ & $\begin{array}{c}\sigma_{3}^{\text {calc }}, \\
\mu \mathrm{b}\end{array}$ \\
\hline \hline 0.0 & $7 / 2^{-}$ & $23.0 \pm 4.4$ & 37.53 & 19.57 & 26.55 \\
1.224 & $9 / 2^{-}$ & $5.6 \pm 1.5$ & 3.44 & 2.96 & 4.50 \\
1.378 & $3 / 2^{-}$ & $24.9 \pm 4.7$ & 50.63 & 14.74 & 23.15 \\
1.505 & $1 / 2^{-}$ & $16.4 \pm 6.3$ & 33.42 & 8.97 & 13.58 \\
1.690 & $11 / 2^{-}$ & $1.1 \pm 0.5$ & 1.56 & 0.93 & 1.21 \\
1.758 & $3 / 2^{-}$ & $18.5 \pm 4.2$ & 40.15 & 11.16 & 16.00 \\
1.897 & $7 / 2^{-}$ & $7.2 \pm 1.8$ & 12.86 & 5.22 & 6.55 \\
1.920 & $5 / 2^{-}$ & $11.3 \pm 3.1$ & 19.49 & 6.77 & 8.79 \\
2.133 & $5 / 2^{+}$ & $14.4 \pm 4.4$ & 38.32 & 8.43 & 13.36 \\
2.133 & $5 / 2^{-}$ & & & & \\
2.479 & $3 / 2^{-*}$ & $4.9 \pm 2.0$ & 18.02 & 5.52 & 5.85 \\
2.485 & $9 / 2^{-}$ & & & & \\
2.514 & $7 / 2^{-*}$ & & & & \\
2.523 & $13 / 2^{-}$ & $3.0 \pm 1.5$ & 19.02 & 4.15 & 4.51 \\
2.560 & $9 / 2^{-}$ & & & & \\
2.611 & $7 / 2^{-}$ & $3.3 \pm 1.6$ & 24.54 & 6.69 & 7.31 \\
2.615 & $9 / 2^{-*}$ & & & & \\
2.723 & $9 / 2^{-*}$ & & & & \\
2.731 & $3 / 2^{-}$ & $12.0 \pm 4.1$ & 30.1 & 7.13 & 9.89 \\
2.743 & $11 / 2^{-}$ & & & & \\
2.804 & $5 / 2^{-}$ & $9.2 \pm 3.5$ & 30.20 & 8.41 & 8.65 \\
2.879 & $3 / 2^{-}$ & & & & \\
2.981 & $1 / 2^{+}$ & $8.0 \pm 3.0$ & 22.36 & 5.05 & 8.63 \\
2.982 & $5 / 2^{-*}$ & & & & \\
\hline & & & &
\end{tabular}

"The superscripts "exp" and "calc" label, respectively, the experimental and calculated cross-section values; the subscripts "1", "2", and "3" on the latter label the theoretical values corresponding, respectively, to the radiative strength functions in a Lorentzian form, to the radiative strength functions calculated with allowance for only the nuclear temperature, and to the radiative strength functions calculated with allowance for nuclear temperature and shell effects. Asterisks indicate spinparity assignments chosen in the present study.

In these expressions and in those that precede them, all values are given in $\mathrm{MeV}$.

The parameters of the real part of the optical potential differ only slightly from the global parameter set that is presented in [8] and which was derived on the basis of data on the scattering of protons with energies in excess of $9 \mathrm{MeV}$; however, the parameters of the imaginary part of the same potential differ from those in the global set more pronouncedly. At the same time, our parameters comply well with the results reported in [10], where an optical-model version that takes into account the dispersion relation between the imaginary and the real part of the potential underlies the description of proton scattering on ${ }^{56} \mathrm{Fe}$ target nuclei at incident-proton energies between 4.08 and $7.74 \mathrm{MeV}$.

The level densities in the ${ }^{57} \mathrm{Co}$ and ${ }^{59} \mathrm{Co}$ nuclei were calculated on the basis of the back-shifted Fermi gas model, with the parameters being set to $a=6.4 \mathrm{MeV}^{-1}$ and $\Delta=-0.02 \mathrm{MeV}$ for the former and to $a=5.5$ 
TABLE II: $\left(p, \gamma_{f}\right)$ partial cross sections for ${ }^{58} \mathrm{Fe}$ targets

\begin{tabular}{|c|c|c|c|c|c|}
\hline$E_{f}, \mathrm{MeV}$ & $J^{\pi}$ & $\begin{array}{c}\sigma^{e x p}, \mu \mathrm{b} \\
E=2.8 \mathrm{MeV} \\
\Delta=220 \mathrm{keV}\end{array}$ & $\begin{array}{c}\sigma_{1}^{c a l c}, \\
\mu \mathrm{b}\end{array}$ & $\begin{array}{c}\sigma_{2}^{\text {calc }} \\
\mu \mathrm{b}\end{array}$ & $\begin{array}{c}\sigma_{3}^{\text {calc }}, \\
\mu \mathrm{b}\end{array}$ \\
\hline 0.0 & $7 / 2^{-}$ & $49.0 \pm 3.9$ & 110.80 & 51.38 & 52.15 \\
\hline 1.099 & $3 / 2^{-}$ & $68.6 \pm 6.4$ & 170.62 & 57.71 & 74.10 \\
\hline 1.190 & $9 / 2^{-}$ & $8.5 \pm 5.0$ & 17.72 & 6.96 & 7.72 \\
\hline 1.292 & $3 / 2^{-}$ & $64.3 \pm 9.7$ & 153.47 & 51.65 & 68.93 \\
\hline 1.434 & $1 / 2^{-}$ & & & & \\
\hline 1.460 & $11 / 2^{-}$ & $65.8 \pm 15$ & 172.14 & 61.78 & 68.74 \\
\hline 1.482 & $5 / 2^{-}$ & & & & \\
\hline 1.745 & $7 / 2^{-}$ & $19.5 \pm 4.3$ & 43.93 & 16.33 & 23.73 \\
\hline 2.062 & $7 / 2^{-}$ & & & & \\
\hline 2.087 & $5 / 2^{-}$ & $29.5 \pm 8.3$ & 87.56 & 32.41 & 46.41 \\
\hline & $9 / 2^{-}$ & $9.5 \pm 2.3$ & 12.17 & 6.42 & 8.03 \\
\hline 2.184 & $11 / 2^{-}$ & & & & \\
\hline 2.205 & $5 / 2^{-}$ & $23.7 \pm 7.4$ & 47.52 & 17.05 & 24.78 \\
\hline 395 & $9 / 2^{-}$ & $4.4 \pm 2.0$ & 9.44 & 3.68 & 4.48 \\
\hline 2.479 & $5 / 2^{-}$ & $19.3 \pm 4.4$ & 40.50 & 14.48 & 20.32 \\
\hline & $5 / 2^{-}$ & & & & \\
\hline 582 & $3 / 2^{-}$ & $68.1 \pm 17$ & 123.86 & 46.65 & 64.52 \\
\hline & $7 / 2^{-}$ & & & & \\
\hline & $1 / 2^{-}$ & & & & \\
\hline 2.722 & $9 / 2^{-}$ & & & & \\
\hline 770 & $3 / 2^{-}$ & $99.9 \pm 25$ & 148.38 & 58.73 & 89.18 \\
\hline & $5 / 2^{-}$ & & & & \\
\hline 2.817 & $3 / 2^{-}$ & $32.9 \pm 8.7$ & 86.43 & 24.47 & 35.45 \\
\hline & $7 / 2^{-}$ & & & & \\
\hline & $3 / 2^{-}$ & & & & \\
\hline 2.958 & $5 / 2^{-}$ & $30.6 \pm 17$ & 147.37 & 42.74 & 54.93 \\
\hline & $3 / 2^{-}$ & & & & \\
\hline 15 & $7 / 2^{-}$ & $4.7 \pm 2.2$ & 20.35 & 7.33 & 9.09 \\
\hline 3.063 & $1 / 2^{-}$ & & & & \\
\hline 3.082 & $9 / 2^{-}$ & $16.7 \pm 6.4$ & 30.33 & 19.78 & 21.47 \\
\hline & $7 / 2^{-}$ & & & & \\
\hline 3.141 & $7 / 2^{-}$ & $4.2 \pm 1.8$ & 19.36 & 6.72 & 8.06 \\
\hline & $3 / 2^{-}$ & $23.4 \pm 12$ & 56.89 & 21.90 & 25.50 \\
\hline & $5 / 2^{-}$ & & & & \\
\hline 3.220 & $3 / 2^{-}$ & $38.4 \pm 8.6$ & 96.21 & 32.46 & 43.27 \\
\hline & $3 / 2^{-}$ & & & & \\
\hline 3.323 & $7 / 2^{-}$ & $4.8 \pm 1.4$ & 17.16 & 5.9 & 6.80 \\
\hline
\end{tabular}

$\mathrm{MeV}^{-1}$ and $\Delta=-0.77 \mathrm{MeV}$ for the latter. In these calculations, we used the rigid-body value for the ${ }^{57} \mathrm{Co}$ moment of inertia and half of it for the ${ }^{59} \mathrm{Co}$ moment of inertia. These parameter sets ensure the best agreement of the computed values of the level densities (see Fig.2) with data from [14,15] on the discrete section of the energy-level diagram for the nuclei being investigated and with data obtained from an analysis of the experimental spectra of neutrons from $(p, n)$ reactions in the proton energy range $E_{p}=6 \div 10 \mathrm{MeV}$ [16,17], as well as with data deduced from an analysis of Ericson fluctuations at $U=14 \mathrm{MeV}$ for ${ }^{57} \mathrm{Co}[18]$.

The scheme used here to compute radiative strength functions [6] takes into account the dependence of the spread width of the giant dipole resonance on the width of the giant dipole resonance on the energy $E_{\gamma}$, the effect

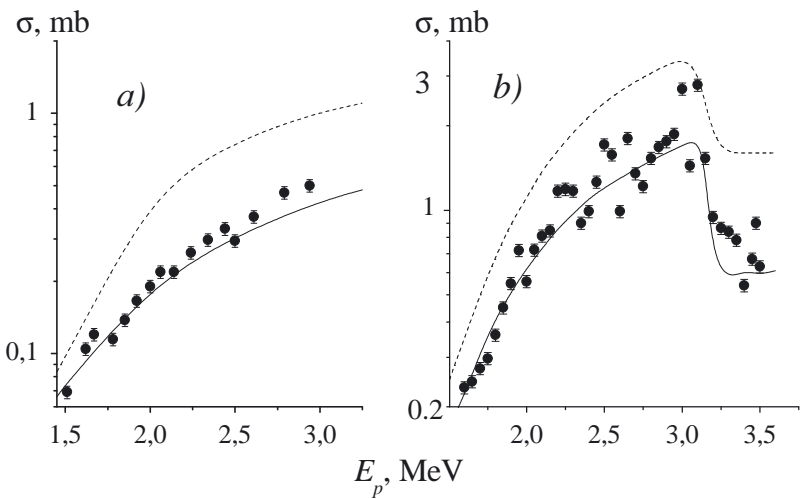

FIG. 4: Total cross sections for the $(p, \gamma)$ reactions on $(a)$ ${ }^{56} \mathrm{Fe}$ and $(b){ }^{58} \mathrm{Fe}$ target nuclei: (closed circles in Fig. $4 a$ ) experimental cross-section values derived from the estimates of the results presented in [11, 12], (closed circles in Fig. 4b) experimental cross-section values from [13], (dashed curves) cross sections computed with the radiative strength functions in a Lorentzian form, and (solid curves) cross sections computed with the radiative strength functions as obtained within the statistical approach developed in $[6,7]$.

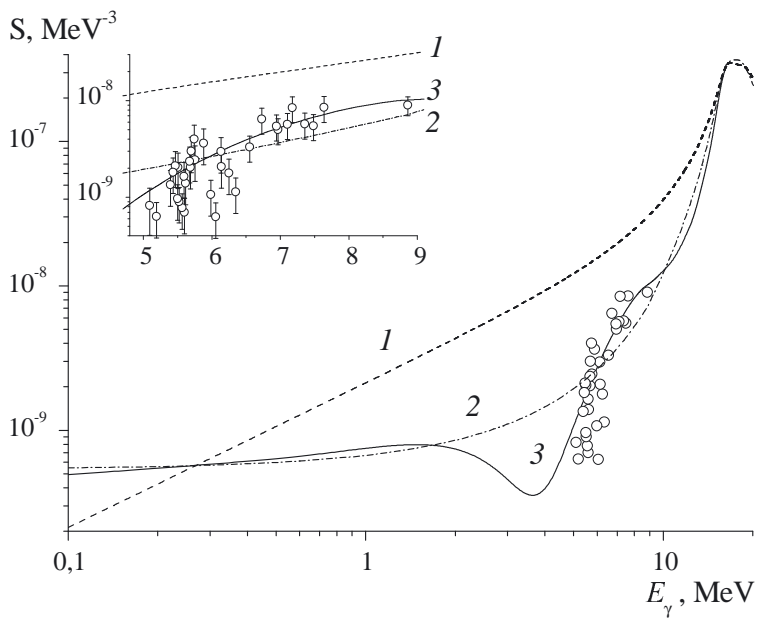

FIG. 5: Experimental and theoretical values of the radiative strength functions for primary gamma transitions in ${ }^{57} \mathrm{Co}$ : (open circles) radiative-strength-function values at $E_{p}=2.84$ $\mathrm{MeV}$, (curve 1) Lorentzian dependence, (curve 2) results of the calculations within the statistical approach with allowance for only nuclear temperature, and (curve 3) results of the calculations within the statistical approach with allowance for the nuclear temperature and shell effects.

of the nuclear temperature, and the role of shell corrections and of the Pauli exclusion principle. Within this approach, the $E 1$ strength function for the case of a doublepeaked giant dipole resonance can be represented in the form

$$
S_{\gamma}^{E 1}=8.674 \cdot 10^{-8} \times 2 \pi\left[1+\exp \left(-E_{\gamma} / T\right)\right]^{-1} \times
$$




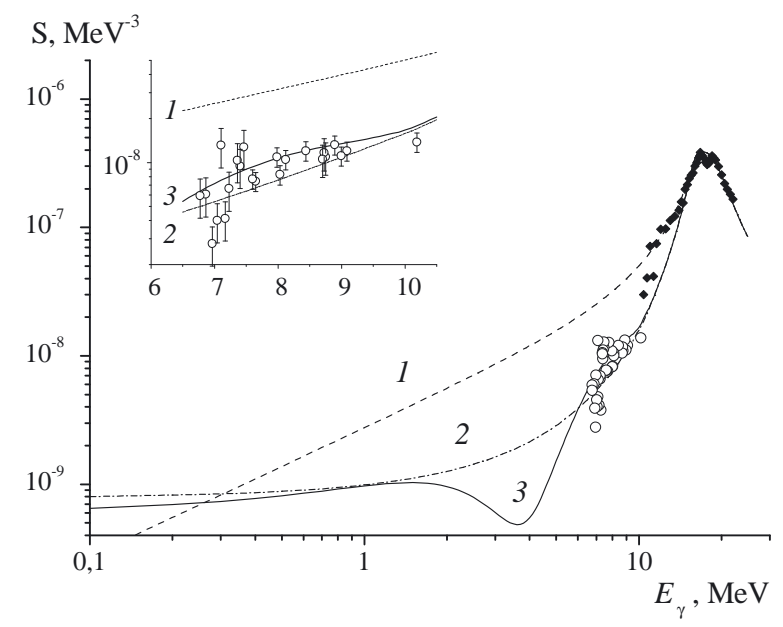

FIG. 6: As in Fig. 5, but for ${ }^{59}$ Co. Open circles represent the radiative-strength-function values at $E_{p}=2.82 \mathrm{MeV}$. Closed diamonds illustrate data from [21]. The notation for the curves is identical to that in Fig. 5.

$$
\times \sum_{i=1}^{2} \frac{\sigma_{i} E_{i}^{2} \Gamma_{R(i)}\left(E_{\gamma}\right)}{\left(E_{\gamma}^{2}-E_{i}^{2}\right)^{2}+E_{i} \Gamma_{R(i)}\left(E_{\gamma}\right)} .
$$

where

$$
\Gamma_{R(i)}\left(E_{\gamma}\right)=\Gamma_{i} \rho_{2 p-2 h}\left(E_{\gamma}, T\right) / \rho_{2 p-2 h}\left(E_{i}, T\right) .
$$

In these expressions, $\sigma, E_{i}$, and $\Gamma_{i}$ are, respectively, the cross sections at the maxima, the positions of the components of the giant dipole resonance, and their widths. The values of these parameters are chosen in such a way as to ensure the best fit of the resulting Lorentzian shape to experimental data that Alvarez et al. [21] present for the $(\gamma, n)$ reaction on ${ }^{59} \mathrm{Co}$. In calculating the level density $\rho_{2 p-2 h}\left(E_{\gamma}, T\right)$, we took into account the shell structure of the spectrum of single-particle levels and the effect of the nuclear temperature on the occupation numbers for these nuclei.

In order to calculate the $M 1$ strength function, we made use of the relation [7]

$$
S_{\gamma}^{E 1} / S_{\gamma}^{M 1}=0.03 A\left(E_{\gamma}^{2}+(\pi T)^{2}\right) / B_{n}^{2},
$$

where $B_{n}$ is the neutron binding energy.

Tables 1 and 2 display the measured values of $\left(p, \gamma_{f}\right)$ partial cross sections for ${ }^{56} \mathrm{Fe}$ and ${ }^{58} \mathrm{Fe}$ target nuclei and the values calculated for these cross sections by formula
(1) with various radiative strength functions. In order to visualize these results more clearly, the experimental and the calculated values of the $\left(p, \gamma_{f}\right)$ partial cross sections for direct gamma transitions to ${ }^{59} \mathrm{Co}$ states are shown in Fig. 3 as a histogram. The shaded areas of the histogram correspond to the cross sections computed with the radiative strength functions found within the statistical approach $[6,7]$ with allowance for the shell structure and nuclear temperature, while the unshaded areas represent cross sections evaluated with the radiative strength functions having a Lorentzian form. In Fig. 4, the theoretical values of the total cross sections for the $(p, \gamma)$ reactions on ${ }^{56} \mathrm{Fe}$ and ${ }^{58} \mathrm{Fe}$ nuclei are contrasted against relevant experimental data.

The radiative-strength-function values obtained in the present study from an analysis of the $(p, \gamma)$ reactions on ${ }^{56} \mathrm{Fe}$ and ${ }^{58} \mathrm{Fe}$ nuclei are displayed in Figs. 5 and 6 . The curves in these figures represent theoretical estimates of the radiative strength function that correspond to a Lorentzian form (curve 1), to the results of the calculations that are based on expression (3) and which allow for only temperature (curve 2), and to the results of analogous calculations including both temperature and shell effects (curve 3). The contribution of .1 transitions, which is not shown in the figures, does not exceed $15 \%$ for various states of ${ }^{57} \mathrm{Co}$ and ${ }^{59} \mathrm{Co}$. That the known values of the giant-dipole-resonance parameters for ${ }^{59} \mathrm{Co}$ were used throughout for want of experimental data on the giant dipole resonance in ${ }^{57} \mathrm{Co}$ obviously had an adverse effect on the degree of agreement between the results of our calculations and the experimental values of the radiative strength functions for ${ }^{57} \mathrm{Co}$.

\section{CONCLUSION}

Our results indicate that, in the gamma-transitionenergy range under study, the absolute values of the radiative strength functions for ${ }^{57} \mathrm{Co}$ and ${ }^{59} \mathrm{Co}$ fall significantly short of values on the Lorentzian curves that describe the corresponding giant dipole resonances. At the same time, the radiative strength functions as calculated within the approach developed in $[6,7]$ this approach relies on Fermi liquid theory and takes into account nuclear temperature and shell effectsagree with experimental data without the use of adjustable parameters. This is at odds with the Brink hypothesis, according to which the radiative strength function for dipole transitions must not depend on the properties of the final nuclear state.
[1] K. Nilson, B. Erlandsson, and A. Marcinkowski, Nucl. Phys. A 391, 61 (1982).

[2] B. Erlandsson, K. Nilson, and A. Marcinkowski, Z. Phys. A 293, 43 (1979).

[3] C. E. Porter and R. G. Thomas, Phys. Rev. 104, 483
(1956).

[4] B. A. Nemashkalo, V. E. Storizhko, and K. V. Shebeko, Yad. Fiz. 36, 1083 (1982) [Sov. J. Nucl. Phys. 36, 633 (1982)].

[5] J. W. Tepel et al., Phys. Lett. B 49, 1 (1974). 
[6] V. K. Sirotkin, Yad. Fiz. 43, 570 (1986) [Sov. J. Nucl. Phys. 43, 362 (1986)].

[7] . V. K. Sirotkin and D. F. Zaretski, Izv. Akad. Nauk SSSR, Ser. Fiz. 52, 984 (1988).

[8] F. D. Becchetti and G. W. Greenlees, Phys. Rev. 182, 1190 (1969).

[9] S. Kailas and M. K. Mehta, in Proceedings of the 2nd IndoUS Symposium on Nucl. Phys. Cyclotron and Intermediate Energy, Bombay, 1982, Vol. 1, p.505.

[10] N. Boukhorouba et al., Phys. Rev. C 46, 2375 (1992).

[11] G. A. Krivonosov et al., Yad. Fiz. 24, 40 (1976) [Sov. J. Nucl. Phys. 24, 239 (1976)].

[12] D. B. Nichols, R. G. Arns, H. J. Hausman, et al., Phys.
Rev. 183, 945 (1969).

[13] S. G. Times et al., Nucl. Phys. A 563, 473 (1993).

[14] M. R. Bhat, Nucl. Data Sheets 67, 195 (1992).

[15] C. M. Baglin, Nucl. Data Sheets 69, 733 (1993).

[16] V. Mishra et al., Phys. Rev. C 49, 750 (1994).

[17] M. I. Svirin and G. N. Smirenkin, Yad. Fiz. 48, 682 (1988) [Sov. J. Nucl. Phys. 48, 437 (1988)].

[18] T. Ericson et al., Annu. Rev. Nucl. Sci. 16, 183 (1966).

[19] M. Kicinska-Habior et al., Phys. Rev. C 36, 612 (1987).

[20] W. Dilg et al., Nucl. Phys. A 217, 269 (1973).

[21] R. A. lvarez et al., Phys. Rev. C 20, 128 (1979). 